\title{
Welcome to the year 2021
}

\author{
Nadia Magnenat-Thalmann ${ }^{1}$
}

Accepted: 16 October 2020

(c) Springer-Verlag GmbH Germany, part of Springer Nature 2020

We are very happy to announce that the Visual Computer Journal has progressed significantly during the year 2020 although we had a hard time due to the Covid19. We are so glad to inform you that the activity of the journal has continued as usual even with the pandemic going on.

During 2020, we have received more than 950 submissions and published more than 180 papers resulting in less than 20 per cent of acceptance rate.

We are also happy to inform you that the impact factor has increased to 1.456 .

We take this opportunity to thank our Associate Editors and Reviewers for their tremendous work in reviewing and handling each paper carefully.

Throughout 2020, some Associate Editors have left the editorial board of the Visual Computer. We would like to thank them for their great work and contribution. They are:

- Jianfei Cai, Nanyang Technological University, Singapore

- Kun Zhou, Zhejiang University, China

- LiangLiang Cao, University of Massachusetts Amherst, USA

- Ofir Weber, Bar-Ilan University, Israel

- Jia Li, Yahoo Inc., USA

- Christian Richardt, University of Bath, UK

- Richard Yi Da Xu, University of Technology Sydney, Australia

- Holly Rushmeier, Yale University, USA

The editorial board of the Visual Computer is renewed regularly. This year, due to the increase of submissions of papers, we have invited several experts in different fields to join the editorial board. We welcome our new Associate Editors who are listed below:

- Bin Fan, Chinese Academy of Sciences, Beijing, China

Nadia Magnenat-Thalmann

thalmann@miralab.ch

1 MIRALab-CUI, University of Geneva, Battelle, Building A, 7, Route de Drize, 1227 Carouge, Geneva, Switzerland
- Dan Xu, Hong Kong University of Science and Technology (HKUST), China

- Daniel Aliaga, Purdue University, USA

- Fanman Meng, University of Electronic Science and Technology of China, China

- Frank Guan, Singapore Institute of Technology, Singapore

- Guanbin Li, Sun Yat-Sen University, China

- Huamin Wang, The Ohio State University, USA

- Hongyuan Zhu, A*STAR, Singapore

- Jie Chen, Hong Kong Baptist University, China

- Quanzeng You, Microsoft Azure \& AI, USA

- Shuhang Gu, ETH Zurich, Switzerland

- Tianjia Shao, Zhejiang University, China

- Xiaodan Liang, Sun Yat-Sen University, China

- Zhaopeng Cui, ETH Zurich, Switzerland

- Pietro Zanuttigh, University of Padova, Italy

We also welcome Jutta Daum who joined the Visual Computer during the year as Project coordinator.

During 2020, the journal published a special issue containing the best papers from the Computer Graphics International Conference (CGI'2020) organized by the Computer Graphics Society (CGS). It was scheduled to be in Geneva, but due to the pandemic, was finally held online from 20 to 23 of October.

Finally, this journal could not exist without the strong support and collaboration of many people. We would like to thank several key persons at Springer who are supporting us continuously, in particular our senior editor, Rachel Moriarty, her senior editorial assistant, Annette Hinze and Beate Uhl, project coordinator who unfortunately had to leave during the year. We are also grateful to Murugeswari Rangasamy for answering and dealing with the daily questions we receive from our authors and reviewers. We would like to thank all of them for their continuous great assistance needed for a journal that handles 12 issues and publishes more than 180 papers a year.

Lastly, we thank all our authors who have submitted their work to the Visual Computer as well as our readers. Without them, the journal could not exist. 
We look forward to 2021 as an exciting new year for the Visual Computer, and we encourage authors to submit the best of their work. We hope that the pandemic will end soon and allow us to organize CGI2021 Conference in September in Geneva.

We wish everyone to stay safe and healthy.
Nadia Magnenat Thalmann

Editor-in-Chief

The Visual Computer

Publisher's Note Springer Nature remains neutral with regard to jurisdictional claims in published maps and institutional affiliations. 